\title{
Simulation of the Effect of Centrifugation on Membrane Efficiency by Using Computational Fluid Dynamics During the Clarification of Pomegranate Juice
}

\author{
Asieh Aghaei ${ }^{1}$, Hossein Mirsaeedghazi ${ }^{2 *}$, Yousef Ramezan ${ }^{1}$ \\ 1. Department of Food Sciences and Technology, Faculty of Advanced Sciences and Technology, Pharmaceutical Sciences Branch, Islamic Azad \\ University, Tehran, Iran. \\ 2. Department of Food Technology, College of Abouraihan, University of Tehran, Pakdasht, Iran.
}

\section{A B S T R A C T}

Background and Objectives: Pomegranate juice is prone to turbidity, which makes it hard to preserve and concentrate. Microfiltration is one of the membrane processes that can be used to reduce its turbidity, but, it is prone to membrane fouling. Pretreatment is considered the most effective way to reduce membrane fouling.

Materials and Methods: This study simulated the effect of centrifugation on membrane clarification during the filtration of pomegranate juice using computational fluid dynamics. The geometry of the membrane unit was plotted in GAMBIT software and meshed with quad-mapped meshes, and the problem was solved using fluent software. Samples were centrifuged at 2000 and $4000 \mathrm{rpm}$ for 5 and $10 \mathrm{~min}$.

Results: The results of simulation showed that centrifugation at $2000 \mathrm{rpm}$ for $10 \mathrm{~min}$ had the highest permeate flux. One the other hand, centrifugation at $4000 \mathrm{rpm}$ had the lowest permeate flux. The experimental results were in agreement with the simulation results.

Conclusions: Velocity of centrifugation should be controlled to maintain the optimum level and achieve the best results. In this study centrifugation at $2000 \mathrm{rpm}$ improved the process yield.

Keywords: Centrifugation, CFD, Clarification, Membrane, Pomegranate, Simulation

\section{Introduction}

Pomegranate, a fruit that originated in the Middle East, has become popular because of its functionality and nutraceutical properties (1). Current research on the health benefits of pomegranate-juice consumption have found that it reduces blood pressure, protects against prostate cancer and arthritis, inhibits cancercell proliferation, has anti-inflammatory effects, stops diarrhea and aids in the treatment of diabetes $(2,3)$. Other studies have proven that pomegranate juice contains much higher levels of antioxidant compounds than other fruit juices and beverages; for example, it has almost three times the amount of either red wine or green tea $(3,4)$. These positive effects were due to high content of polyphenolic compounds. The phenolic constituents of pomegranates play an important role in the sensory properties of the juice, such as color, bitterness and astringency.

However, during juice processing (concentration and storage), polymerization or condensation of these polyphenols can lead to polymeric complexes creation between some components such as metal ions, sugars, polysaccharides and proteins. To prevent the development of sediment and haze during storage, the juice must be clarified (4).

Microfiltration that works by pressure driving force can be useful to clarify pomegranate juice $(5,6)$. All membrane-based separation processes are subject to membrane fouling; one of the most effective ways to reduce membrane fouling is pretreatment $(5,7)$. Of the available pretreatment methods, centrifugation is the most efficient, as it costs less than enzymatic 
processes and does not introduce additives into the juice (8). Computational fluid dynamics (CFD), can be used to develop a model with widespread applicability in membrane technology; however, there have been few attempts to model membrane systems using CFD (9). The main advantage of a CFD analysis is that it offers the ability to predict the permeate flux for complex geometries and to improve the design to attain higher flux. Mirsaeedghazi et al. used CFD to predict velocity and pressure patterns in the membrane module during the clarification of pomegranate juice. These results suggest that feed flow must be parallel to the membrane surface at the entrance of the module to reduce the pressure created in vertical patterns, thus reducing the likelihood of damage to the membrane (6). Sharifanfar et al. used CFD to simulate the effect of feed-canal height on the efficiency of the membrane clarification of pomegranate juice and found that permeate volume was directly dependent on feed-canal height (10). Rei et al. studied the efficacy of various pretreatment processes in comparison to pectinase treatment to determine whether the addition of pectinase could be substituted for juice pretreatment (8).

Research has shown that that the cost of pretreatment by centrifugation is approximately four times less than that of enzymatic treatment, based on a steady state of permeate flux (8). Chhaya et al. compared the performance of centrifugation versus microfiltration as a primary clarification process for stevia extract, finding that the performance of centrifugation was marginally better (11).

To date no study has used CFD to simulate the effect of centrifugation on the performance of membrane treatment of pomegranate juice. In the current study, the effect of centrifugation on the permeation flux during membrane treatment of pomegranate juice was simulated with CFD.

\section{Materials and Methods}

Geometry setting in GAMBIT: The study used a flat sheet module $130 \mathrm{~mm}$ long and $24 \mathrm{~mm}$ wide (Fig. 1). The geometry of module was planned using GAMBIT (version 2.4.6) in a cross-flow pattern. The side walls were meshed (with a space of 0.769 ) at double-sided form with ratio equal to 1.2 in lower side. The membrane surface and the upper and lower walls were meshed in single-sided form with different ratios and spacing. The meshes had Quad-type elements (Fig. 1). All of these parameters were determined by trial and error, with the goal being to avoid the conflict between the cells. The boundary conditions at A, B and D points (Fig. 1) were velocity inlet, pressure outlet and pressure outlet, respectively. The membrane was assumed to be a porous jump and the wall was selected for all other dimensions. Final pattern was used to solve in FLUENT 6.3.26.
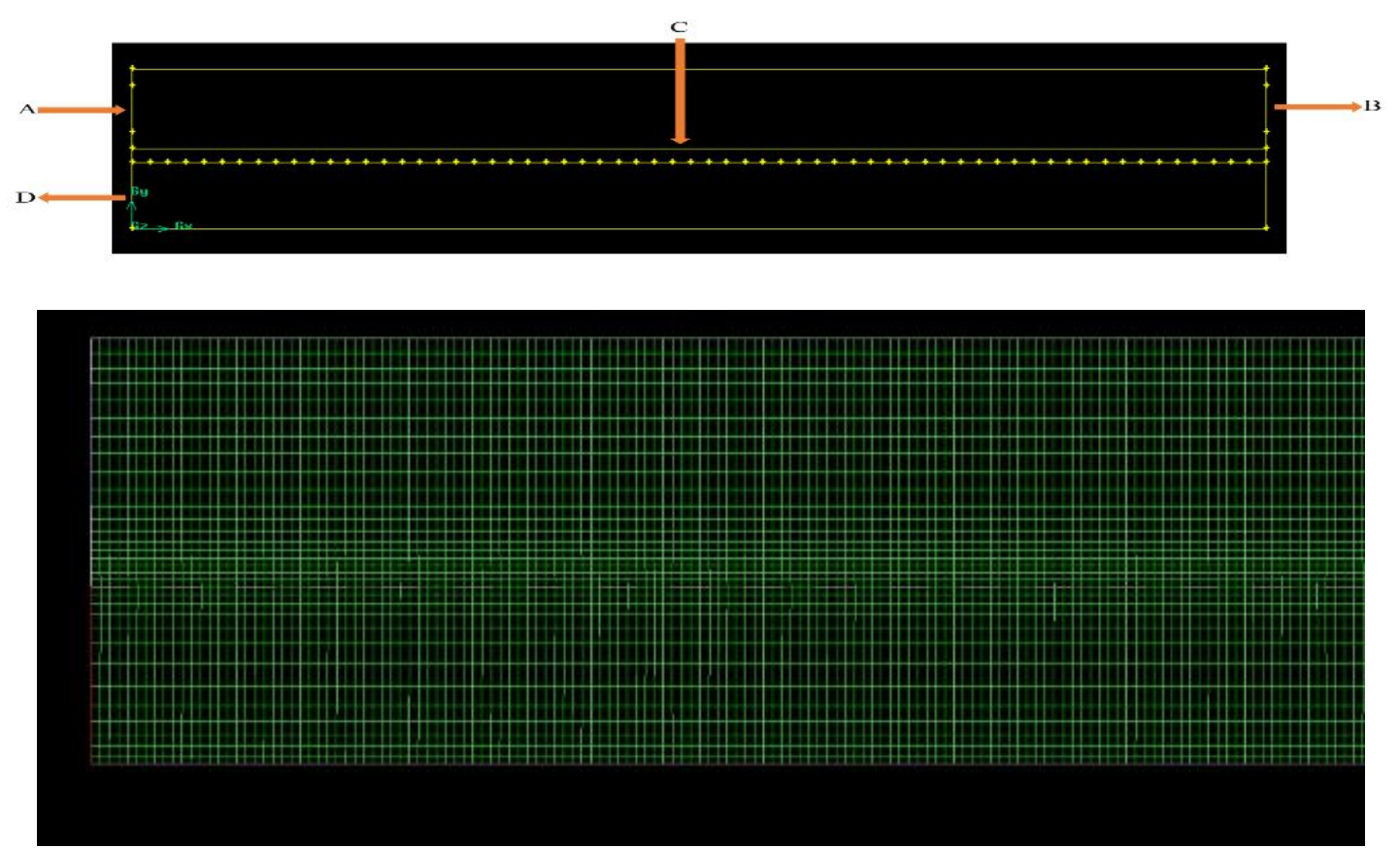

Fig. 1. Geometry and a view of mesh structure (A: feed inlet, B: retentate outlet, C: membrane, D: permeate outlet) 
Solution method in FLUENT: A two-dimensional double-precision procedure was selected in the FLUENT software. The selected solver was segregated, with an implicit formulation at the steady state. The selected material was pomegranate juice, and the density and viscosity of each sample were adjusted as shown in Table 1. Gauge pressure was selected for the pressure, and gravity force was activated. The pressures of permeate and retentate were considered as 1 and 0.45 bar, respectively. Feed velocity was adjusted at $0.5 \mathrm{~m} / \mathrm{s}$. The face permeability of the porous jump was calculated as:

$$
\alpha=\frac{D_{p}^{2}}{150} \frac{\varepsilon^{2}}{(1-\varepsilon)^{2}}
$$

Where $\mathrm{D}_{\mathrm{p}}$ and $\varepsilon$ were the average diameter of the pomegranate juice particles and the membrane void, respectively.

The pressure jump was calculated as:

$$
C_{2}=\frac{3.5}{D_{p}} \frac{(1-\varepsilon)}{\varepsilon^{2}}
$$

Table 1. Some physical properties of different treatments

\begin{tabular}{ccccc}
\hline Sample & $\begin{array}{c}\text { Centrifuge } \\
(\mathrm{rpm})\end{array}$ & $\begin{array}{c}\text { Time } \\
(\mathrm{min})\end{array}$ & $\begin{array}{c}\text { Density } \\
(\mathrm{kg} / \mathrm{m} 2)\end{array}$ & $\begin{array}{c}\text { Viscosity } \\
(\mathrm{kg} / \mathrm{ms})\end{array}$ \\
\hline 1 & - & - & 1093 & 0.0027 \\
2 & 2000 & 5 & 1085.5 & 0.00206 \\
3 & 2000 & 10 & 1084 & 0.00208 \\
4 & 4000 & 5 & 1082 & 0.0024 \\
5 & 4000 & 10 & 1082.5 & 0.00247 \\
\hline
\end{tabular}

Membrane unit: The membrane type was mixed cellulose ester (Millipore, USA) with a pore size of $0.22 \mu \mathrm{m}$ and a thickness of $150 \mu \mathrm{m}$. The membrane surface was coated with gold with a sputter coater (BAL-TEC, SCDOOS, Switzerland) using a physical vapor deposition method and then the photos were taken using scanning electron microscopy (SEM) (Philips, XL30, The Netherlands) to calculate the membrane void. Result showed that there was approximately one pore in each $1 \mu \mathrm{m}^{2}$ of membrane surface.

Eq. (3) was used to calculate the permeate flux (J. $\left.\mathrm{kg} / \mathrm{m}^{2} . \mathrm{s}\right)$.

$$
J_{p}=\frac{\Delta m}{A \times t}
$$

Where $\mathrm{A}, \mathrm{m}$, and $\mathrm{t}$ are the membrane surface $\left(\mathrm{m}^{2}\right)$, weight of permeate $(\mathrm{kg})$ and time (s), respectively.

Evaluation of mean particle size: Mean particle size was measured using a particle-size analyzer (Malvern, model Hydro 2000 S, Worcestershire, England); results were analyzed using Mastersizer 2000 software.

Juice extraction: Pomegranates (variety Malase Saveh) were obtained from a local market (Saveh, Iran), washed, and their leathery skins were removed. The sacs were manually pressed and the juice was obtained, and large particles were removed using a mesh filter. Centrifugation of pomegranate juice samples was carried out in a HERMLE Z $300 \mathrm{~K}$ centrifuge. Samples were centrifuged for 5 and 10 $\mathrm{min}$ at 2000 and $4000 \mathrm{rpm}$. The supernatant was used for further procedures and analyses.

\section{Results}

Evaluation of pressure counters showed that in all tests the pressure values at the beginning and end of the upper canal were greater than the value in the center. In contrast, the pressure decreased suddenly in the lower canal (Fig. 2). The pressure also decreased on the surface of the membrane due to membrane permeability. Results showed that the greatest pressure drop was observed in the canal after centrifugation at $2000 \mathrm{rpm}$ for $10 \mathrm{~min}$; these parameters also gave the greatest pressure in the lower canal. The smallest pressure drop in the upper canal and the lowest pressure in the lower canal were observed when no centrifugation process was performed.

Evaluation of the velocity contours in the flatsheet module showed that the velocity in the input section of the feed canal was much greater than its value in the output section (Fig. 3); however, feed velocity was increased on the surface of membrane. On the other hand, the velocity of permeate after feed centrifugation at 2000rpm for $10 \mathrm{~min}$ was much greater than the other parameters. The velocity magnitude at the exit was greatest at the bottom of the permeate outlet valve. The velocity significantly fell at the center of the exit valve, and it slightly increased at the top of the channel (Fig. 4). 


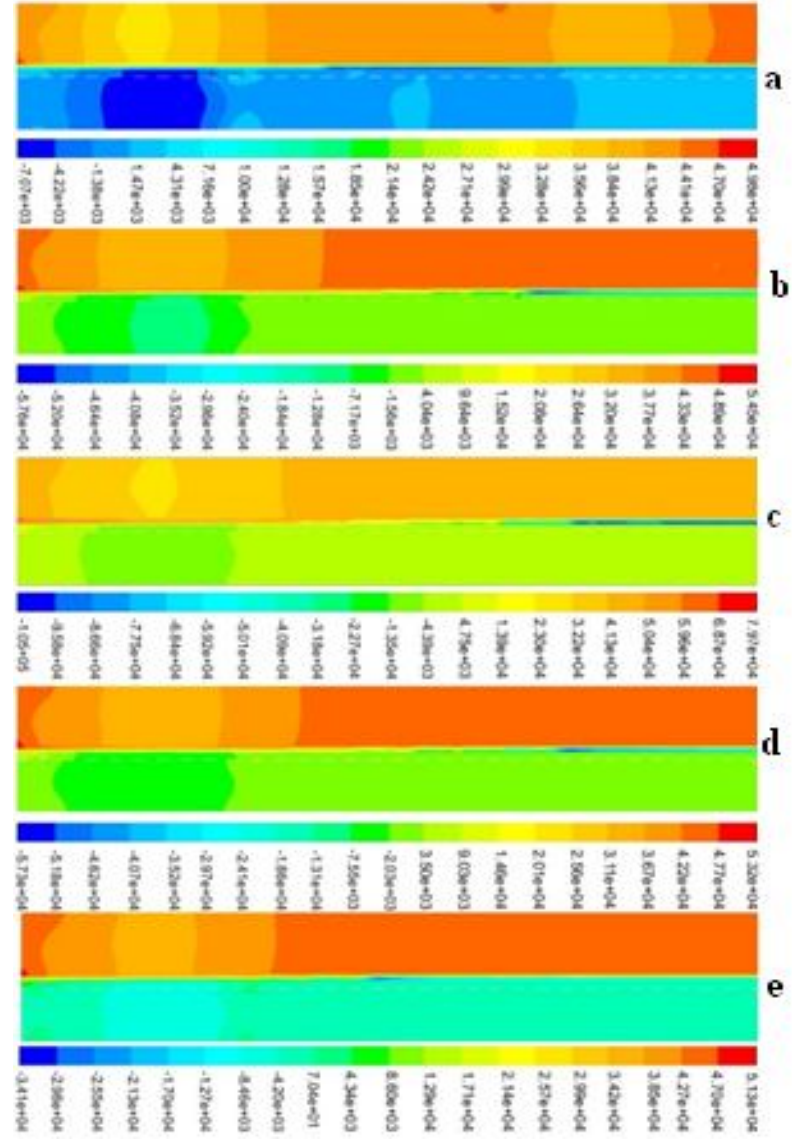

Fig. 2. The contours of pressure in flat sheet membrane module (a: $4000 \mathrm{rpm}, 10 \mathrm{~min}$; b: $4000 \mathrm{rpm}, 5 \mathrm{~min}$; c: 2000 rpm, 10 min; d: 2000 rpm 5 min; e: untreated juice)
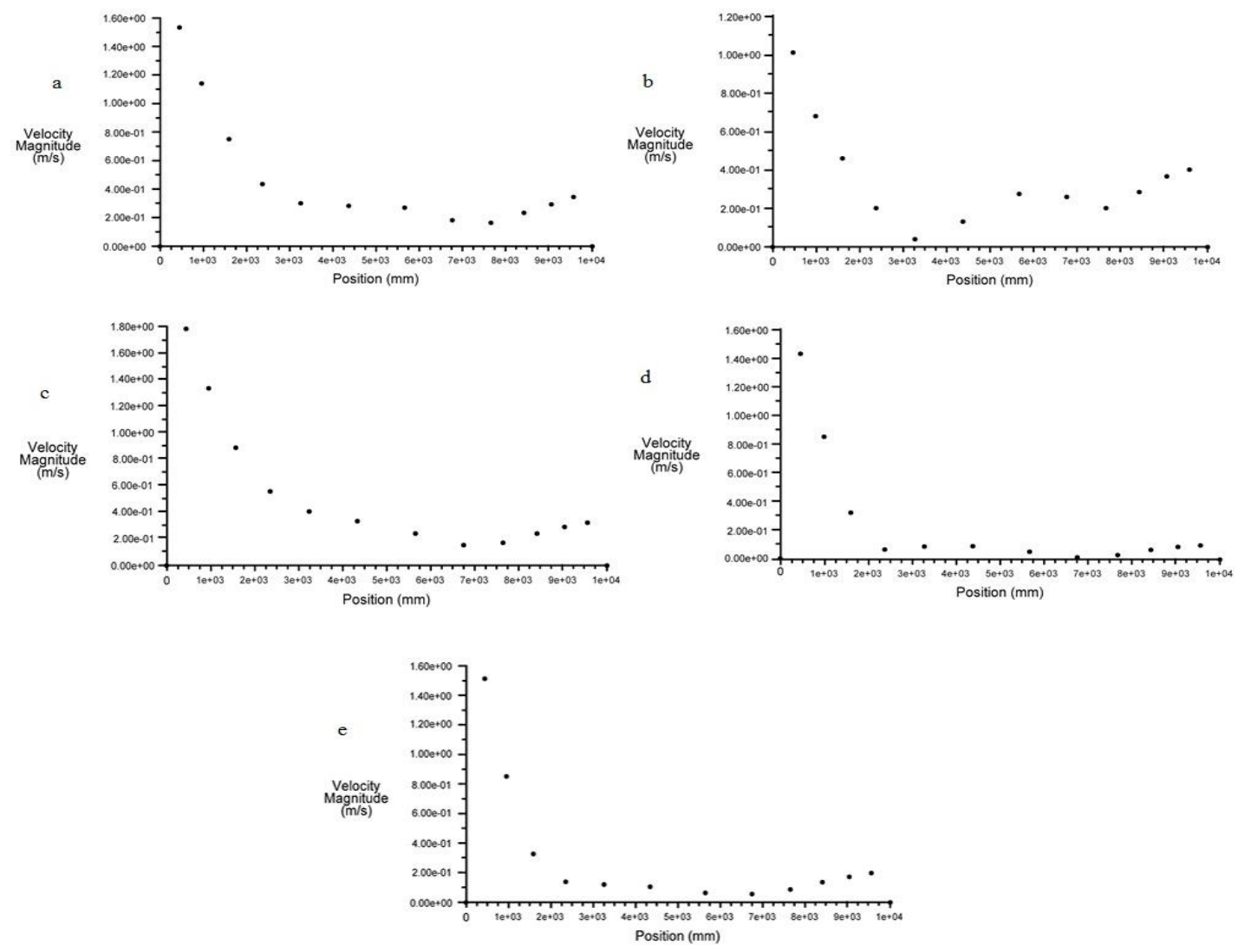

Fig. 4. Vector of velocity at the outlet of permeate in the flat sheet module (a: 2000rpm, 5min; b: $2000 \mathrm{rpm}$, $10 \mathrm{~min}$; c: 4000rpm, 5 min; d: 4000rpm, 10 min; e: untreated juice)

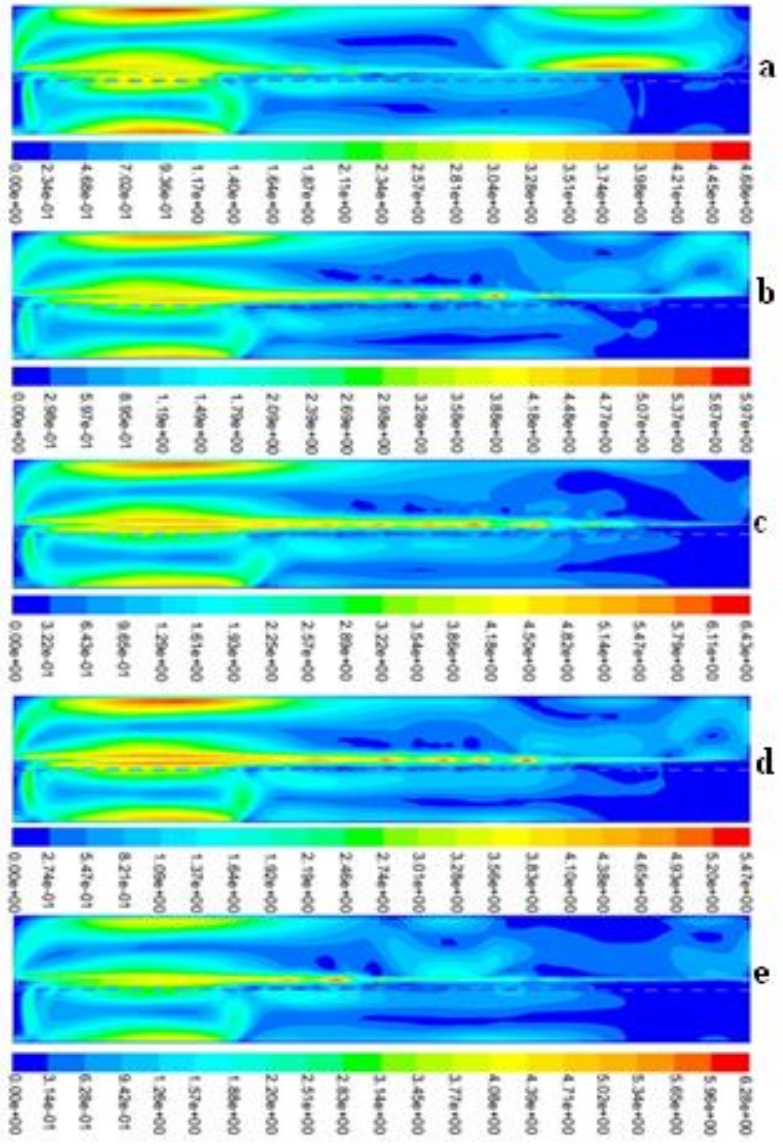

Fig. 3. The velocity pattern in the membrane module (a: $4000 \mathrm{rpm}, 10 \mathrm{~min}$; b: $4000 \mathrm{rpm}, 5 \mathrm{~min}$; : $2000 \mathrm{rpm}, 10$ min; d: 2000 rpm 5 min; e: untreated juice)

Position $(\mathrm{mm})$ 
Pomegranate juice was centrifuged at 2000 and $4000 \mathrm{rpm}$ for 5 and $10 \mathrm{~min}$, then clarified with the membrane unit to verify the results that had been obtained using CFD. Results showed that permeate flux for juice that was centrifuged at $2000 \mathrm{rpm}$ for 10 min was higher than for the other samples throughout the process. Pomegranate juice that was centrifuged at $4000 \mathrm{rpm}$ for $10 \mathrm{~min}$ and untreated pomegranate juice had almost the same behavior; both had the lowest amount of the permeate flux (Fig. 5). These experimental results were in agreement with the results obtained from the simulation.

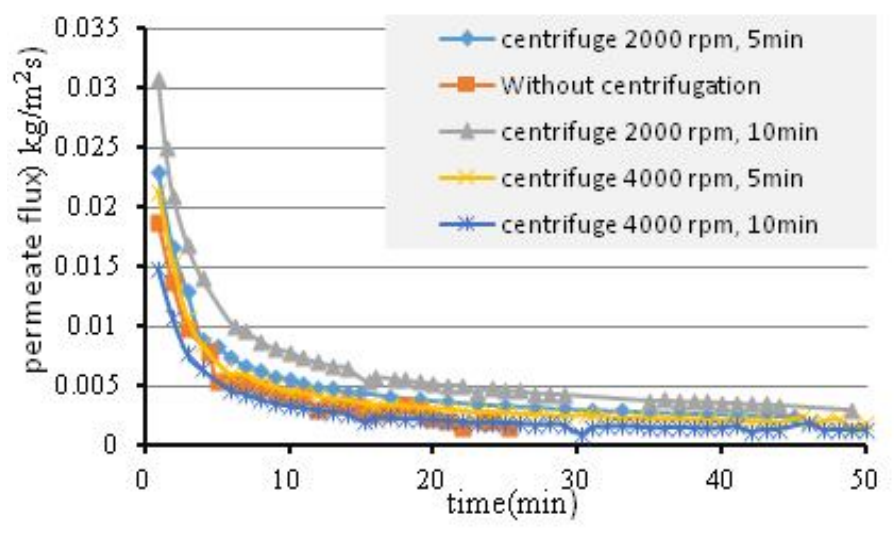

Fig. 5. The permeate flux during different conditions of membrane clarification

\section{Disc ussion}

Increasing the pressure at the beginning and end of the upper canal was due to the effects of the pump and exit valve, respectively. Also, sudden reduction of the pressure in lower canal was due to the barrier effect of the membrane between the upper and lower canals.

Bigger velocity in the input of feed canal comparing output section was probably due to separation of part of the feed as permeate over the membrane surface. Increasing the feed velocity on the surface of membrane was due to the existence of pressure toward membrane surface and the barrier effect of the membrane against the feed-flow direction. The velocity magnitude at the exit was due to the direction of the fluid stream, which was toward the left side of the channel after passing through the membrane, and the effect of gravity, which would cause the accumulation of mass permeate at the bottom of the channel. According to previous findings, the mean value for velocity in the exit valve was at its maximum after feed centrifugation at 2000 rpm for $10 \mathrm{~min}$. This indicates the greatest penetration of feed through the membrane.

Evaluation of pressure counters showed that the results were according to the findings of some other researchers. Mirsaeedghazi et al. resulted that feed pressure over the membrane surface in entrance of membrane module was much higher than other sections leading to membrane rupturing (6). Sharifanfar et al. studied the effect of feed canal height on the efficiency of membrane clarification of pomegranate juice. They found similar results on the velocity change profile in the feed canal. On the other hand, our results showed that increasing the centrifugation velocity did not improve the process performance (10). Domingues et al. found similar results in the clarification of passion fruits juice with centrifugation and enzymatic pretreatments (12).

\section{Conclusion}

Centrifugation improved the permeate flux in the membrane clarification of pomegranate juice due to reduction of the large particles including cellulose, pectin and phenolic compounds. Reduction of large particles minimizes fouling, improving membrane efficiency. However, centrifugation as a pretreatment method has different effects on the efficiency of membrane clarification of pomegranate juice depending on its time and velocity. Simulation of the effect of centrifugation on the efficiency of membrane clarification of pomegranate juice showed that this pretreatment at $2000 \mathrm{rpm}$ improved the process yield; however, increasing the centrifugation velocity to $4000 \mathrm{rpm}$ had the reverse effect. Hence, centrifugation of pomegranate juice before membrane clarification has both positive and negative effects on process performance, and its velocity should be controlled to stay at the optimum level.

\section{Financial disclosure}

The authors declared no financial interest.

\section{Funding/Support}

There is no funding.

\section{References}

1. Suzanne D, Johanningsmeier G, Harris K, Pomegranate as a functional food and nutraceutical source. J Food Sci Technol 2011; 2: 181-201.

2. Bagci PO. Effective clarification of pomegranate juice: A comparative study of pretreatment methods and their influence on ultrafiltration flux. Int J Food Eng 2014; 141: 58-64. 
3. Baklouti S, Ellouze-Ghorbel R, Mokni A, Chaabouni S. Clarification of pomegranate juice by ultrafiltration: study of juice quality and of the fouling mechanism. J Fruits 2011; 67: 215-225.

4. Onsekizoglu P, Production of high quality clarified pomegranate juice concentrate by membrane processes. J Membrane Sci 2013; 442: 264-271.

5. Mirsaeedghazi H, Emam-Djomeh Z, Mousavi SM, Aroujalian A, Navidbakhsh M, Changes in blocking mechanisms during membrane processing of pomegranate juice. Int J Food Sci Technol 2009; 44: 2135-2141.

6. Mirsaeedghazi H, Emam-Djomeh Z, Mousavi SM, Aroujalian A, Navidbakhsh M, Modelling the membrane clarification of pomegranate juice with computational fluid dynamics. J Eur Food Res Technol 2011; 232: 671-677.

7. Mohanty K, Purkait MK, Membrane technologies and applications. Taylor \& Francis 2012.
8. Rai P, Majumdar GC, Jayanti VK, Dasgupta S, De S, Alternative pretreatment methods to enzymatic treatment for clarification of mosambi juice using ultrafiltration. J Food Process Eng 2006; 29: 202-218.

9. Paipuri M, Hassan O, Numerical Modelling of Membrane Filtration using Lattice Boltzmann and Finite Volume Methods. Swansea University 2014.

10. Sharifanfar R, Mirsaeedghazi H, Fadavi A, Kianmehr $\mathrm{MH}$, Evaluation of the effect of feed canal height on membrane clarification efficiency of pomegranate juice using computational fluid dynamics (CFD). J Desalination Water Treat 2016; 57: 1-7.

11. Chhaya, Majumdar GC, De S, Primary clarification of stevia extract: a comparison between centrifugation and microfiltration. J Sep Sci Technol 2013; 48: 113-121.

12. Domingues RCC, Faria Junior SB, Silva RB, Cardoso VL, Reis MHM, Clarification of passion fruit juice with chitosan: Effects of coagulation process variables and comparison with centrifugation and enzymatic treatments. Process Biochem 2012; 47(3): 467-71. 